\title{
Lack of high-mass pre-stellar cores in the starless MDCs of NGC $6334^{\star}$
}

\author{
F. Louvet ${ }^{1}$, S. Neupane ${ }^{1}$, G. Garay ${ }^{1}$, D. Russeil ${ }^{2}$, A. Zavagno ${ }^{2}$, A. Guzman ${ }^{1}$, L. Gomez ${ }^{3}$, L. Bronfman ${ }^{1}$, and T. Nony ${ }^{4}$ \\ ${ }^{1}$ Universidad de Chile, Camino el Observatorio 1515, Las Condes, Santiago de Chile, Chile \\ e-mail: flouvet@das.uchile.cl \\ ${ }^{2}$ CNRS, LAM, Laboratoire d'Astrophysique de Marseille, Aix-Marseille Université, Marseille, France \\ 3 Joint ALMA Observatory, Alonso de Córdova 3107, Vitacura, Santiago, Chile \\ ${ }^{4}$ IPAG, Université Grenoble Alpes, 38000 Grenoble, France
}

Received 12 November 2017 / Accepted 31 October 2018

\begin{abstract}
Context. The formation of high-mass stars remains unknown in many aspects. There are two competing families of models to explain the formation of high-mass stars. On the one hand, quasi-static models predict the existence of high-mass pre-stellar cores sustained by a high degree of turbulence. On the other hand, competitive accretion models predict that high-mass proto-stellar cores evolve from low or intermediate mass proto-stellar cores in dynamic environments.

Aims. The aim of the present work is to bring observational constraints at the scale of high-mass cores ( $\sim 0.03 \mathrm{pc})$.

Methods. We targeted with ALMA and MOPRA a sample of nine starless massive dense cores (MDCs) discovered in a recent Herschel/HOBYS study. Their mass and size $\left(\sim 110 M_{\odot}\right.$ and $r=0.1 \mathrm{pc}$, respectively) are similar to the initial conditions used in the quasi-static family of models explaining for the formation of high-mass stars. We present ALMA 1.4 mm continuum observations that resolve the Jeans length $\left(\lambda_{\text {Jeans }} \sim 0.03 \mathrm{pc}\right)$ and that are sensitive to the Jeans mass $\left(M_{\text {Jeans }} \sim 0.65 M_{\odot}\right)$ in the nine starless MDCs, together with ALMA- ${ }^{12} \mathrm{CO}(2-1)$ emission line observations. We also present $\mathrm{HCO}^{+}(1-0), \mathrm{H}^{13} \mathrm{CO}^{+}(1-0)$ and $\mathrm{N}_{2} \mathrm{H}^{+}(1-0)$ molecular lines from the MOPRA telescope for eight of the nine MDCs.

Results. The nine starless MDCs have the mass reservoir to form high-mass stars according to the criteria by Baldeschi et al. (2017). Three of the starless MDCs are subvirialized with $\alpha_{\mathrm{vir}} \sim 0.35$, and four MDCs show sign of collapse from their molecular emission lines. ALMA observations show very little fragmentation within the MDCs. Only two of the starless MDCs host compact continuum sources, whose fluxes correspond to $<3 M_{\odot}$ fragments. Therefore, the mass reservoir of the MDCs has not yet been accreted onto compact objects, and most of the emission is filtered out by the interferometer.

Conclusions. These observations do not support the quasi-static models for high-mass star formation since no high-mass pre-stellar core is found in NGC 6334. The competitive accretion models, on the other hand, predict a level of fragmentation much higher than what we observe.
\end{abstract}

Key words. stars: formation - stars: massive - ISM: clouds - ISM: structure - methods: observational - techniques: interferometric

\section{Introduction}

Two main theoretical scenarios have been proposed to explain the formation of high-mass stars: (1) monolithic collapse of a massive dense core $\left(\mathrm{MDC}, \sim 100 M_{\odot}\right.$ for a radius of $\sim 0.1 \mathrm{pc}$ ) supported by supersonic turbulent pressure (e.g. McKee \& Tan 2002; Krumholz et al. 2007) or (2) competitive accretion in a proto-cluster environment through Bondi-Hoyle accretion (e.g. Bonnell et al. 2004; Bonnell \& Bate 2006). These two scenarios lead to distinct characteristics for the initial stages of high-mass star formation. The first family of models suppose the existence of starless MDCs that are supported by high-degrees of micro-turbulence ( $\sigma \sim 1.7-2 \mathrm{~km} \mathrm{~s}^{-1}$; Krumholz et al. 2007). The MDCs contract quasi-statically to become high-mass pre-stellar cores $\left(\sim 30 M_{\odot}\right.$ for a radius of $\left.\sim 0.03 \mathrm{pc}\right)$ before becoming proto-stellar cores. Here, "core" refers to a gaseous structure of $\sim 0.03$ pc (Bontemps et al. 2010; Zhang et al. 2014a; Palau et al. 2015) that will collapse to form a single star or a

\footnotetext{
* MOPRA data presented in the article and a copy of Tables 1 , 2, A.1 and A.2 are only available at the CDS via anonymous ftp to cdsarc.u-strasbg.fr $(130.79 .128 .5)$ or via http://cdsarc.u-strasbg.fr/viz-bin/qcat?J/A+A/622/A99
}

small N-tuple binary system. Hence, quasi-static models predict the existence of one, or a few, high-mass pre-stellar cores in the starless MDCs.

In the second family of models, high-mass pre-stellar cores never develop. The starless MDCs fragment into a cluster of low-mass cores with initial masses of the order of the Jeans mass. The protostars inside these cores start accreting gas from their envelope, unimpeded by the presence of other protostars. In a second step, the gas is accreted from regions outside of their envelopes and therefore from region that can contribute mass to more than one protostar. This accretion accounts for the bulk of the mass of the more massive stars. The latter are formed more favourably at the centre of the MDCs, where the gas density is the highest.

A few high-resolution studies have been performed with (sub)millimeter interferometers with the aim of identifying prestellar cores and protostars within massive dense cores (MDCs; see Rathborne et al. 2007; Swift 2009; Zhang et al. 2009; Busquet et al. 2010; Bontemps et al. 2010; Pillai et al. 2011; Wang et al. 2011, 2014; Zhang \& Wang 2011; Beuther et al. 2013; Lee et al. 2013; Tan et al. 2013; Louvet et al. 2014; Cyganowski et al. 2014; Duarte-Cabral et al. 2014; Fontani et al. 2016; Kong et al. 2017; Palau et al. 2018; Nony et al. 2018). 
Table 1. Physical parameters of the MDCs.

\begin{tabular}{|c|c|c|c|c|c|c|c|c|c|c|c|c|c|}
\hline $\begin{array}{c}\text { MDC } \\
(1) \\
\end{array}$ & $\begin{array}{c}\text { Radius } \\
\text { (pc) } \\
(2) \\
\end{array}$ & $\begin{array}{l}\text { Mass } \\
\left(M_{\odot}\right) \\
(3) \\
\end{array}$ & $\begin{array}{c}v_{\mathrm{N}_{2} \mathrm{H}^{+}} \\
\left(\mathrm{km} \mathrm{s}^{-1}\right) \\
(4)\end{array}$ & $\begin{array}{c}\sigma_{\mathrm{N}_{2} \mathrm{H}^{+}} \\
\left(\mathrm{km} \mathrm{s}^{-1}\right) \\
(5)\end{array}$ & $\begin{array}{c}v_{\mathrm{HCO}^{+}} \\
\left(\mathrm{km} \mathrm{s}^{-1}\right) \\
(6)\end{array}$ & $\begin{array}{c}v_{\mathrm{H}^{13} \mathrm{CO}^{+}} \\
\left(\mathrm{km} \mathrm{s}^{-1}\right) \\
(7)\end{array}$ & $\begin{array}{c}\delta V \\
- \\
(8) \\
\end{array}$ & $\begin{array}{c}M_{\mathrm{vir}} \\
\left(M_{\odot}\right) \\
(9) \\
\end{array}$ & $\begin{array}{c}\alpha_{\mathrm{vir}} \\
- \\
(10) \\
\end{array}$ & $\begin{array}{c}M_{\mathrm{thr}} \\
\left(M_{\odot}\right) \\
(11) \\
\end{array}$ & $\begin{array}{c}M_{\text {Jeans }} \\
\left(M_{\odot}\right) \\
(12) \\
\end{array}$ & $\begin{array}{c}\lambda_{\text {Jeans }} \\
(\mathrm{mpc}) \\
(13)\end{array}$ & $\begin{array}{c}B_{\text {field }} \\
(\mathrm{mG}) \\
(14) \\
\end{array}$ \\
\hline NGC 6334-MDC\#11 & 0.140 & 170 & - & - & -1.8 & - & - & - & - & 63.7 & 0.85 & 46 & 0.7 \\
\hline NGC 6334-MDC\#16 & 0.075 & 150 & -4.0 & 1.00 & -5.6 & -3.7 & -1.6 & 60 & 0.40 & 27.8 & 0.21 & 15 & 2.2 \\
\hline NGC 6334-MDC\#17 & 0.050 & 150 & -7.3 & 1.72 & -7.8 & - & -0.3 & 120 & 0.80 & 16.2 & 0.25 & 11 & - \\
\hline NGC 6334-MDC\#21 & 0.135 & 130 & -4.9 & 0.99 & -4.6 & -4.7 & +0.3 & 107 & 0.80 & 85.7 & 1.31 & 55 & - \\
\hline NGC 6334-MDC\#27 & 0.070 & 105 & -5.4 & 0.77 & -5.5 & -5.4 & -0.1 & 34 & 0.30 & 25.3 & 0.42 & 21 & 1.9 \\
\hline NGC 6334-MDC\#26 & 0.040 & 100 & -5.3 & 1.60 & -6.3 & -4.8 & -0.6 & 83 & 0.80 & 12.0 & 0.28 & 10 & - \\
\hline NGC 6334-MDC\#35 & 0.105 & 90 & -1.9 & 0.65 & -2.0 & - & -0.2 & 36 & 0.35 & 43.4 & 0.93 & 42 & 0.7 \\
\hline NGC 6334-MDC\#44 & 0.150 & 77 & - & - & - & - & - & - & - & 69.8 & 1.15 & 68 & - \\
\hline NGC 6334-MDC\#45 & 0.055 & 76 & -8.9 & 1.12 & -9.2 & -8.9 & -0.3 & 56 & 0.75 & 16.4 & 0.34 & 17 & 0.4 \\
\hline Mean & 0.090 & 116 & & 1.12 & & & & 71 & 0.60 & 40.0 & 0.64 & 32 & 1.2 \\
\hline
\end{tabular}

These attempts revealed either that they were proto-stellar in nature when observed at high resolution (e.g. Duarte-Cabral et al. 2013), or filled with low-mass pre-stellar cores (e.g. Tan et al. 2013; Kong et al. 2017). In total, only five high-mass pre-stellar core candidates have been reported:

- The pre-stellar candidate CygX-N53-MM2 ( 25 $M_{\odot}$ within $0.025 \mathrm{pc}$ ) of Duarte-Cabral et al. (2014); however, owing to the confusion with the neighbour CygX-N53-MM1, it is hard to exclude that CygX-N53-MM2 is driving outflows.

- The pre-stellar candidate G11.92-0.61-MM2 (>30M $\odot$ within $0.01 \mathrm{pc}$ ) of Cyganowski et al. (2014) but the lack of (sub)millimeter molecular line emissions casts doubt about its belonging to the Milky Way.

- The pre-stellar candidate G11.11-P6-SMA1 ( $30 M_{\odot}$ within $0.02 \mathrm{pc}$ ) of Wang et al. (2014). This source seems deprived of outflows but a spectral survey of this target is necessary to determine if it hosts a hot core.

- The source G028CA9 ( 70 $M_{\odot}$ within 0.04 pc) of Kong et al. (2017) but this source still lacks of shock tracer analysis to address if it is driving outflows. Also, according to the authors, it shows complex kinematics potentially indicative of two merging structures.

- The source W43-MM1-\#6 ( $55 M_{\odot}$ within 1300 au) of Nony et al. (2018). The target seems deprived of outflows and its spectral survey did not reveal strong complex organic molecule emissions. Nevertheless, the study could not rule out the existence of an unresolved outflow and/or a small and embedded hot core.

Therefore, although many regions have been studied, very few high-mass pre-stellar core candidates have been reported which proves that, if they exist, they are very elusive. Here, we present a study of nine starless MDCs located in NGC 6334.

The source NGC 6334 is a giant molecular cloud complex $\left(\sim 7 \times 10^{5} M_{\odot}\right.$; see e.g. Russeil et al. 2012) of the Milky Way. It belongs to the Sagittarius-Carina arm, lying at $1.75 \mathrm{kpc}$ from the sun (Matthews et al. 2008). It is a very active highmass star-forming region hosting more than 2000 young stellar objects identified with Spitzer (Willis et al. 2013), numerous H II regions, maser sources, and molecular outflows (see Loughran et al. 1986; Persi \& Tapia 2008; Carral et al. 2002). As part of the Herschel/HOBYS project, Tigé et al. (2017) analysed the properties of the MDCs forming in NCG6334, based on Herschel-PACS\&SPIRE, JCMT-SCUBA2, APEXLABOCA, SEST-SIMBA, Spitzer-IRAC\&MIPS, WISE, and MSX data. Among other results, they found and characterized
16 candidate starless massive dense cores that remained undetected at $70 \mu \mathrm{m}$, have a mass $>70 M_{\odot}$, and a deconvolved radius of $\sim 0.1 \mathrm{pc}$. Thirteen of these MDCs were observed with ALMA (2015.1.00850.S, PI: Louvet, F.). In this article we present nine of these MDCs (see Table 1), as the other four contain protostars and will be presented separately (Louvet et al., in prep.). We complement our dataset with MOPRA observations to analyse the large-scale $(\sim 0.1 \mathrm{pc})$ features of the MDCs. The article is organized so that in Sect. 2 we describes the observational data used in the present work, Sects. 3.1-3.2 present the large-scale feature of the MDCs at $\sim 0.1 \mathrm{pc}$ scales, Sect. 3.3 presents the structure of the MDCs down to $\sim 0.006 \mathrm{pc}$ scales with ALMA. In Sect. 4 we discuss our results with respect to the numerical models of high-mass star formation (Sect. 4.1) and with respect to recent interferometric studies (Sect. 4.2). Finally, in Sect. 5 we describe our conclusions.

\section{Observations}

\subsection{ALMA observations}

ALMA observations were carried out in Cycle 3 in Band 6 using 39 antennas with baselines ranging from $15 \mathrm{~m}$ to $459 \mathrm{~m}$, that gives access to an angular resolution of $\sim 0.74^{\prime \prime}$ and a filtering scale of $11.6^{\prime \prime}$ at $1.4 \mathrm{~mm}$. In this article, we only present continuum emission observations and ${ }^{12} \mathrm{CO}(2-1)$ emission line observations. The continuum is obtained from a bandwidth spectral window of $1.875 \mathrm{GHz}$ centred at $214.5 \mathrm{GHz}$ (or $1.40 \mathrm{~mm}$ ) divided in 128 channels of $31.25 \mathrm{MHz}$ each. The ${ }^{12} \mathrm{CO}(2-1)$ molecular line emission is obtained from a spectral windows centred on the ${ }^{12} \mathrm{CO}(2-1)$ transition at $230.53 \mathrm{GHz}$ with a high spectral resolution of $0.485 \mathrm{MHz}$ (or $0.63 \mathrm{~km} \mathrm{~s}^{-1}$ ). Each of our nine sources was observed for $\sim 1.4 \mathrm{~min}$ in total. The system temperature has a mean value of $91 \mathrm{~K}$. The data was pipeline-calibrated using standard procedures of CASA 4.5.2. The continuum was imaged concatenating together the 128 channels of the spectral window dedicated to the continuum emission, which is a valid approach for pre-stellar structures for which no complex organic molecule emission lines are expected (Motte et al. 2018a). The ${ }^{12} \mathrm{CO}(2-1)$ emission line was slightly smoothed to a spectral resolution of $0.7 \mathrm{~km} \mathrm{~s}^{-1}$. Both the continuum and ${ }^{12} \mathrm{CO}$ line emissions were imaged with the Clark method using the weighting Briggs with a robust parameter of $0.5^{1}$. The mean achieved root mean square

\footnotetext{
1 Details on the method can be found in the CASA Task reference manual at the following location: https://casa.nrao.edu/docs/ taskref/tclean-task.html.
} 


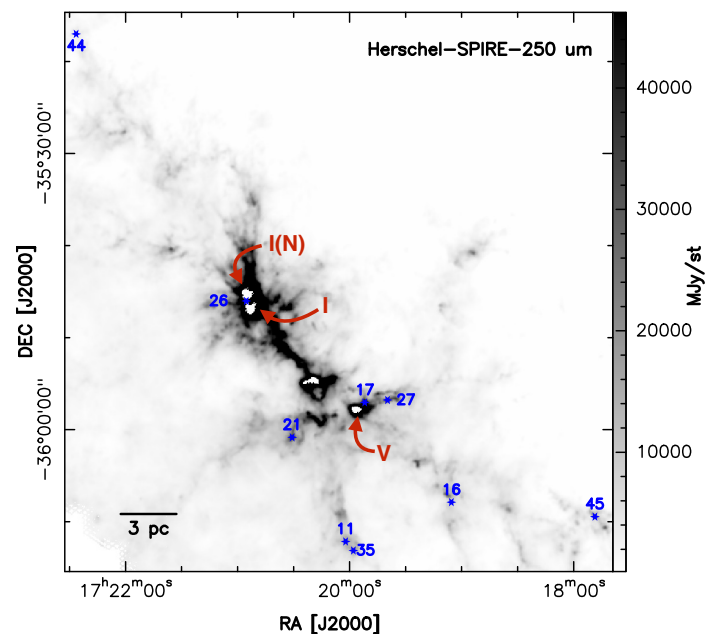

Fig. 1. Herschel-SPIRE-250 $\mu \mathrm{m}$ emission of the NGC 6334 region. The 9 MDCs of the present work are located by the blue stars together with their number, following the nomenclature by Tigé et al. (2017). Red arrows and Roman numerals (I, V) denote bright far-infrared sources from Kraemer \& Jackson (1999). Credits: ESA, Herschel Science Archive.

(rms) noise level is of $\sim 0.34 \mathrm{mJy} / \mathrm{beam}$ in continuum and of $16.6 \mathrm{mJy}$ beam ${ }^{-1}$ per channel in ${ }^{12} \mathrm{CO}(2-1)$, at a resolution of $0.7 \mathrm{~km} \mathrm{~s}^{-1}$. Table A.1 details the rms for each source.

\subsection{MOPRA observations}

These observations were performed in September 2008 with the ATNF MOPRA 22-m telescope with the $3 \mathrm{~mm}$ receiver and the MOPRA spectrometer (MOPS) in the "zoom mode" that allows to observe simultaneously up to 16 different frequencies, among them the $\mathrm{HCO}^{+}(1-0)$ at $89.18 \mathrm{GHz}$, the $\mathrm{H}^{13} \mathrm{CO}^{+}(1-0)$ at $86.75 \mathrm{GHz}$, and the $\mathrm{N}_{2} \mathrm{H}^{+}(1-0)$ at $93.17 \mathrm{GHz}$ emission lines. Small maps (between $3^{\prime} \times 3^{\prime}$ and $5^{\prime} \times 5^{\prime}$ ) were observed in the direction of the 40 dense cores of Russeil et al. (2010). These maps cover seven of our nine MDCs. These maps were done in the OTF mode and the data reduction was performed with Livedata and Gridzilla tools ${ }^{2}$ producing data-cubes with a spectral and spatial resolution of $0.11 \mathrm{~km} \mathrm{~s}^{-1}$ and $30^{\prime \prime}$, respectively. The MOPRA dataset is complemented with observations from the MALT90 survey (Jackson et al. 2013) to cover one additional MDC. To sum up, we gather a coherent sample of spectral lines from the MOPRA telescope for all our nine MDCs but the MDC \#44.

\section{Analysis}

\subsection{Large-scale view of the MDCs}

Figure 1 shows the Herschel $250 \mu \mathrm{m}$ emission of NGC 6334, overshowing the column density map plotted with the positions of the nine MDCs studied here. Figures 2-4 show the column density map over each MDC (left columns) and the molecular line emissions in the MDC (right columns). All observed MDCs are detected in $\mathrm{HCO}^{+}$. Five MDCs are detected in $\mathrm{H}^{13} \mathrm{CO}^{+}$, and seven in $\mathrm{N}_{2} \mathrm{H}^{+}$(see Table 1). Only the MDC \#11 was undetected in both $\mathrm{N}_{2} \mathrm{H}^{+}$and $\mathrm{H}^{13} \mathrm{CO}^{+}$. We fitted the lines in $\mathrm{CLASS}^{3}$ using the $h f s$ procedure to fit the multiplet $\mathrm{N}_{2} \mathrm{H}^{+}$molecular emission

\footnotetext{
2 See http://wwW.atnf.csiro.au/computing/software/index. html.

3 See https: //www . iram. fr/IRAMFR/GILDAS/doc/html/classhtml/class.html.
}

line and Gaussian functions to fit the $\mathrm{HCO}^{+}$and $\mathrm{H}^{13} \mathrm{CO}^{+}$molecular line emissions (see Table 1). We used the fit to the $\mathrm{N}_{2} \mathrm{H}^{+}$ optically thin molecular line to determine the velocity dispersion in the MDCs as $\sigma_{\mathrm{N}_{2} \mathrm{H}^{+}}=\frac{F W H M_{\mathrm{N}_{2} \mathrm{H}^{+}}}{\sqrt{8 \ln 2}}$. We used these velocity dispersion estimates $\sigma_{\mathrm{N}_{2} \mathrm{H}^{+}}{ }^{4}$ to derive the virial mass for each MDC as

$M_{\mathrm{vir}}=3\left(\frac{5-2 n}{3-n}\right) \times \frac{R \sigma_{\mathrm{N}_{2} \mathrm{H}^{+}}}{G}$,

where $R$ is the radius of the MDC, $G$ is the gravitational constant, and $n$ is the index of the density profile $\rho(R) \propto R^{-n}$. Garay et al. (2007) and Mueller et al. (2002) find, on average, a radial profile index of 1.8 for massive dense cores. We use this value of $n=1.8$ to derive the virial mass of our MDCs.

We compute the virial parameter $\alpha_{\mathrm{vir}}=M_{\mathrm{vir}} / M_{\mathrm{MDC}}$, where $M_{\mathrm{MDC}}$ is the mass of the MDC, as reported by Tigé et al. (2017). The MDCs appear either close to virial equilibrium $\left(\alpha_{\mathrm{vir}} \sim 0.8\right.$ for \#17, \#21, \#26, and \#45) or sub-virialized ( $\alpha_{\mathrm{vir}} \sim 0.35$ for \#16, $\# 27$, and \#35). This indicates that they are not transient objects and will most likely collapse and form stars.

\subsection{Infall motion in MDCs}

The majority of the MDCs show a blueshifted central velocity of the optically thick $\mathrm{HCO}^{+}$emission line with respect to the optically thin $\mathrm{H}^{13} \mathrm{CO}^{+}$and/or $\mathrm{N}_{2} \mathrm{H}^{+}$emission lines (see Table 1). Following Mardones et al. (1997), we quantify the asymmetry parameter $\delta V=\left(V_{\text {thick }}-V_{\text {thin }}\right) / \sigma V_{\text {thin }}$ to measure line asymmetry, where $V_{\text {thick }}$ is the peak velocity of the optically thick line, $V_{\text {thin }}$ is the peak velocity of the optically thin line and $\sigma V_{\text {thin }}$ is the line width of the optically thin line. A negative $\delta V$ value is indicative of infall, and a positive value of $\delta V$ is indicative of expansion motions. The typical error in line asymmetry parameter is of 0.25 , which is dominated by the error in determination of velocity of optically thick line. We report one positive value of $\delta V$ (\#MDC 21) and 6 negative values of $\delta V$. The sources \#16, $\# 17$, \#26 and \#45 further have $|\delta V|$ values above the typical error, which strongly indicate infall motions in these MDCs.

Myers et al. (1996) presented a simple model of contracting cloud, and showed that low-velocity contracting cloud display optically thick emission lines with double-peak spectra where the blueshifted peaks appear stronger than the redshifted peaks. At higher infall velocities the redshifted peak of the optically thick emission lines disappear, displaying instead a shoulder-like profile. The four MDCs that have a significant negative $\delta \mathrm{V}$ value display such profiles. Figure 5 presents the optically thick $\mathrm{HCO}^{+}$ emission line superposed with the optically thin $\mathrm{H}^{13} \mathrm{CO}^{+}$emission line $\left(\mathrm{N}_{2} \mathrm{H}^{+}\right.$for \#17) toward these four MDCs. In addition to the blue-shift of the optically thick line with respect to the optically thin line, the optically thick $\mathrm{HCO}^{+}$emission lines display a shoulder-like profile toward the MDCs \#17 and \#26, and a higher blue-peak with self absorption dips in the MDCs \#16, and \#45. Therefore, from the values of the $\delta \mathrm{V}$ parameters together with the profile of their optically thick lines, we conclude that these four MDCs are undergoing infalling motions.

4 Rigorously, the virial mass should be computed from an average molecule of weight $\mu m_{p}$ where $\mu=2.8$ (Kauffmann et al. 2008) and $m_{p}$ is the mass of a proton. Considering the $\mathrm{N}_{2} \mathrm{H}^{+}$as the molecule used to measure the dispersion, it gives $\sigma=\sqrt{\sigma_{\mathrm{N}_{2} \mathrm{H}^{+}}^{2}+\frac{k T}{m_{p}}\left(\frac{1}{2.8}-\frac{1}{29}\right)}$, which differs by less than $5 \%$ with the value of $\sigma_{\mathrm{N}_{2} \mathrm{H}^{+}}$. 

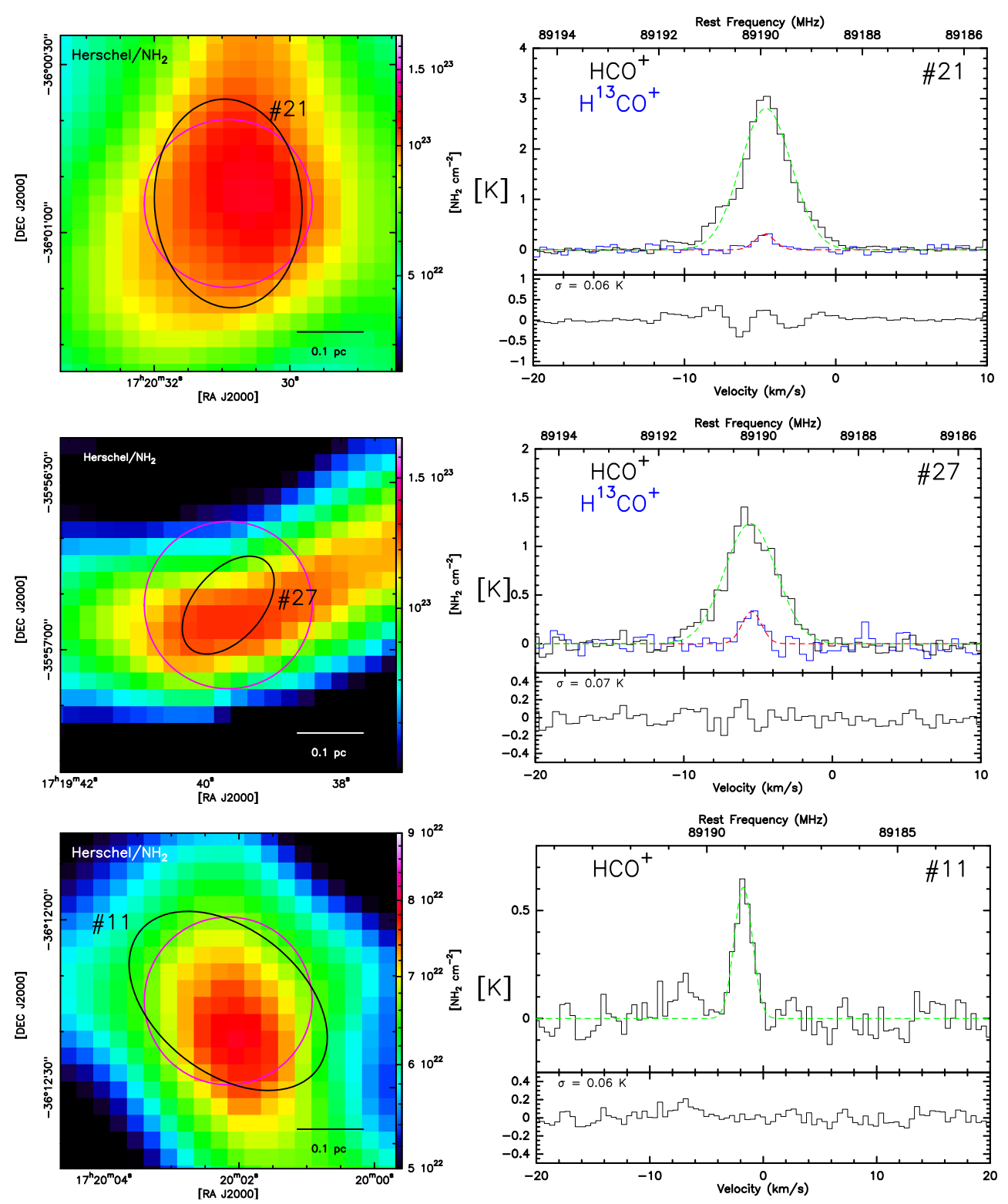

Fig. 2. Left panel: Herschel column density maps toward the nine MDCs studied here. The black ellipse indicates the position and size of the MDC as extracted by Tigé et al. (2017), and the magenta circle shows the beam of the MOPRA telescope. Right panel: $\mathrm{HCO}^{+}(1-0)$ and $\mathrm{H}^{13} \mathrm{CO}^{+}(1-0)$ (or $\mathrm{N}_{2} \mathrm{H}^{+}$, see the label in the panels) emission lines toward the MDCs as seen with MOPRA. The lines are adjusted by a Gaussian (or with the $h f s$ procedure in CLASS to fit the multiplet of the $\mathrm{N}_{2} \mathrm{H}^{+}$) whose results are presented in Table 1 . The frame on the bottom displays the residuals of the $\mathrm{HCO}^{+}(1-0)$ emission after subtraction of the Gaussian feature shown on the top frame.

\subsection{ALMA view of the massive dense cores}

Figures 6-8 present ALMA continuum $1.4 \mathrm{~mm}$ emission maps toward the nine starless MDCs. The emission is completely resolved out in the MDCs \#11, \#17, \#21, \#27, \#35, and \#44, indicating that they are emitting at a scale near or larger than our filtering scale of $11.6^{\prime \prime}$ (or $\sim 0.1 \mathrm{pc}$ at the adopted distance of NGC 6334). On the contrary, the maps toward the MDCs $\# 16$, \#26, and \#45 display some structure. To extract the compact sources within the fields we used the source extraction tool Getsources (Men'shchikov et al. 2012). Developed for multiwavelength Herschel images, it calculates the local noise and local background to properly extract compact sources from a complex cloud environment (see e.g. Louvet et al. 2014). In total we find eight cores located in the vicinity of the MDCs $\# 16, \# 26$, and \#45. In these three fields, we superposed contours showing the column density emission derived from the Herschel observations (respectively the JCMT/SCUBA-2/450 $\mu \mathrm{m}$ emission for the MDC\#26). In the pointing toward the MDC \#16, two fragments are within the area corresponding to $90 \%$ of the column density peak emission of the MDC. Therefore, these two fragments are likely to have formed from the mass reservoir of the MDC\#16. In a similar way, one fragment observed with ALMA seems associated with the MDC\#26. On the other hand, the five remaining fragments are offset from the MDC locations, and generally associated with proto-stellar objects previously identified by Tigé et al. (2017). The integrated flux of these cores varies between 1 and $15 \mathrm{mJy}$. To derive the corresponding mass, we used the expression:

$$
M=\frac{S_{1.4 \mathrm{~mm}}^{\mathrm{int}} \times d^{2}}{\kappa_{1.4 \mathrm{~mm}} \times B_{1.4 \mathrm{~mm}}},
$$

with $d=1.75 \mathrm{kpc}$, and $B_{1.4 \mathrm{~mm}}$ the Planck function at the temperature $T_{\text {dust }}$ given in Table A.1. The dust mass opacity was taken to be equal to $\kappa_{1.4 \mathrm{~mm}}=9.9 \times 10^{-3} \mathrm{~cm}^{2} \mathrm{~g}^{-1}$, following the $\kappa_{v}=0.1 \mathrm{~cm}^{2} \mathrm{~g}^{-1} \times(v / 1000 \mathrm{GHz})^{\beta}$ equation including a gas to dust ratio of 100 and an opacity index $\beta=1.5$, which is typical of dense and cool media (Ossenkopf \& Henning 1994). The mass of the cores ranges between 0.2 and $2.6 M_{\odot}$ for a mean size of $\sim 0.008 \mathrm{pc}$. Compared with the mass of the MDCs $\left(\sim 115 M_{\odot}\right)$, it is clear that most of the emission is resolved out even in these MDCs where substructure is found. The parameters of each low-mass core are reported in Table 2. 

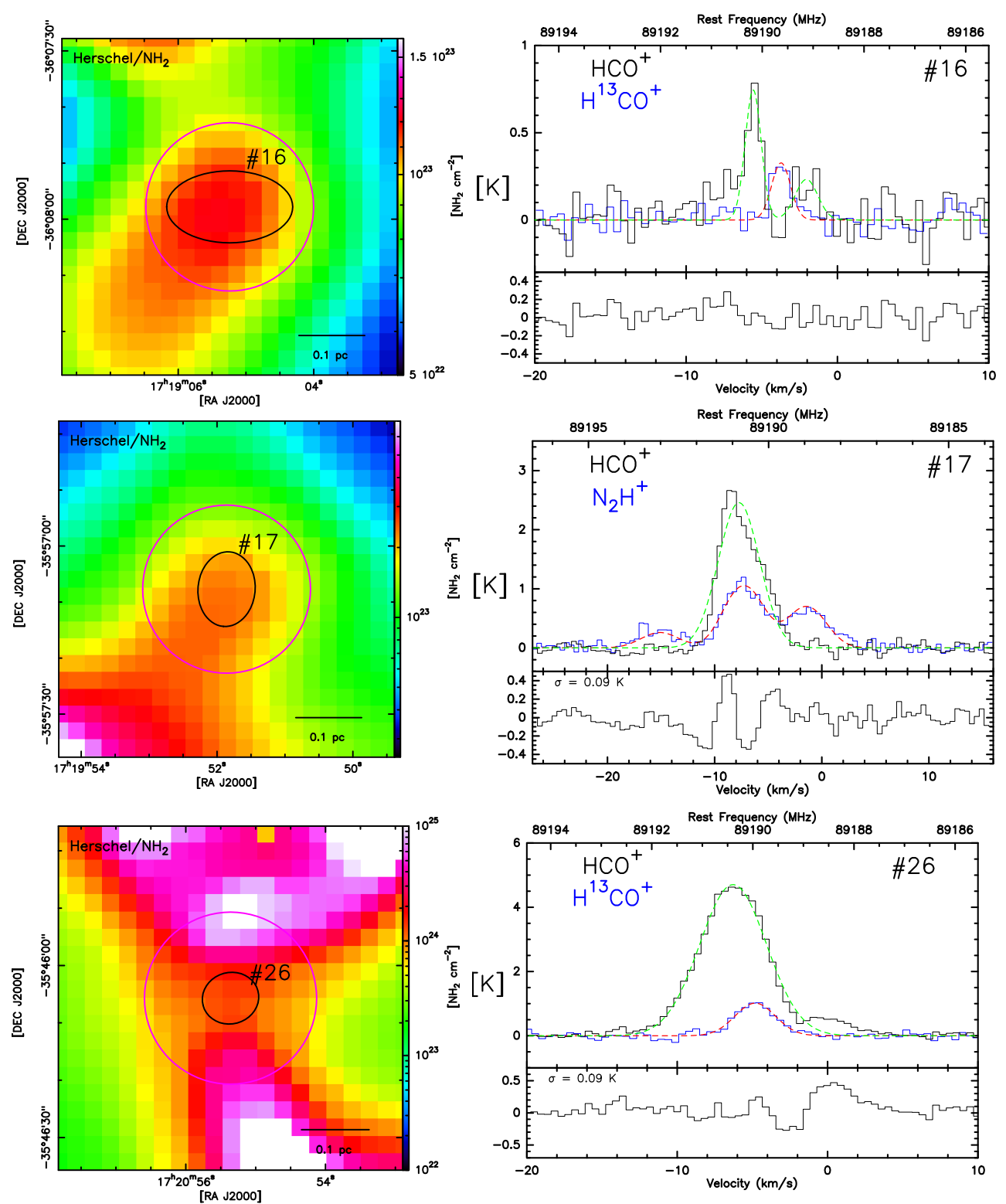

Fig. 3. Same as Fig. 2, for the MDCs $\# 16$, \#17, and \#26.

The blue and red contours in Figs. 6-8 show the blueshifted and redshifted integrated emissions of the ${ }^{12} \mathrm{CO}(2-1)$, highlighting the presence of outflows. The three compact sources \#16-alma1, \#16-alma2, \#26-alma38 are deprived of outflows and are therefore pre-stellar core candidates. We report the detection of a bipolar outflow associated with the proto-stellar object \#45-alma1. We also report two ${ }^{12} \mathrm{CO}$ blueshifted blobs, plus one ${ }^{12} \mathrm{CO}$ redshifted blob in the MDC \#11; and one elongated ${ }^{12} \mathrm{CO}$ redshifted emission in the MDC \#35. These ${ }^{12} \mathrm{CO}$ emissions are not associated with any compact continuum sources, down to our detection limit of $0.11 M_{\odot}$ corresponding to a $5 \sigma$ level. The interpretation of these features goes beyond the scope of the present paper and will require the addition of complementary data, for example, from ACA, to recover the intermediate scale emission.

\section{Discussion}

\subsection{Comparison with models}

Baldeschi et al. (2017) find an empirical high-mass star formation threshold, based on studies of environment with and without high-mass star formation. They suggest that cloud structures with masses larger than the mass $m_{\text {threshold }}=1280 M_{\odot}(r / p c)^{1.42}$, where $r$ is the source radius, form high-mass stars or will form high-mass stars in the near future. Applying this relationship to our $\sim 0.1 \mathrm{pc}$ MDCs, we find that all have masses above the mass threshold for high-mass star formation (see Table 1, column 11).

Our nine MDCs have a mean mass of $\sim 115 M_{\odot}$ and a mean radius of $0.1 \mathrm{pc}$. These conditions are very similar with the initial conditions of the quasi-static models for high-mass star formation (100-200 $M_{\odot}$ and radius of $0.1 \mathrm{pc}$; see e.g. McKee \& Tan 2002; Krumholz et al. 2007). Three of the starless MDCs are clearly subvirialized with $\alpha_{\text {vir }} \sim 0.35$, and three additional MDCs display evidence of infall from their large-scale molecular line emissions, in contrast with the virial equilibrium assumption used in these models. In the framework of quasi-static models, where the MDCs $(r \sim 0.1 \mathrm{pc})$ slowly contract down to the physical scale at which individual (or low N-tuple system) star formation takes place $(\sim 0.03 \mathrm{pc}$; see e.g. Bontemps et al. 2010; Zhang et al. 2014b; Palau et al. 2015), one expects to observe high-mass pre-stellar cores. Nevertheless, the ALMA observations toward NGC 6334 show that the few cores that were detected are low-mass cores (maximum mass of $2.6 M_{\odot}$ ), and 

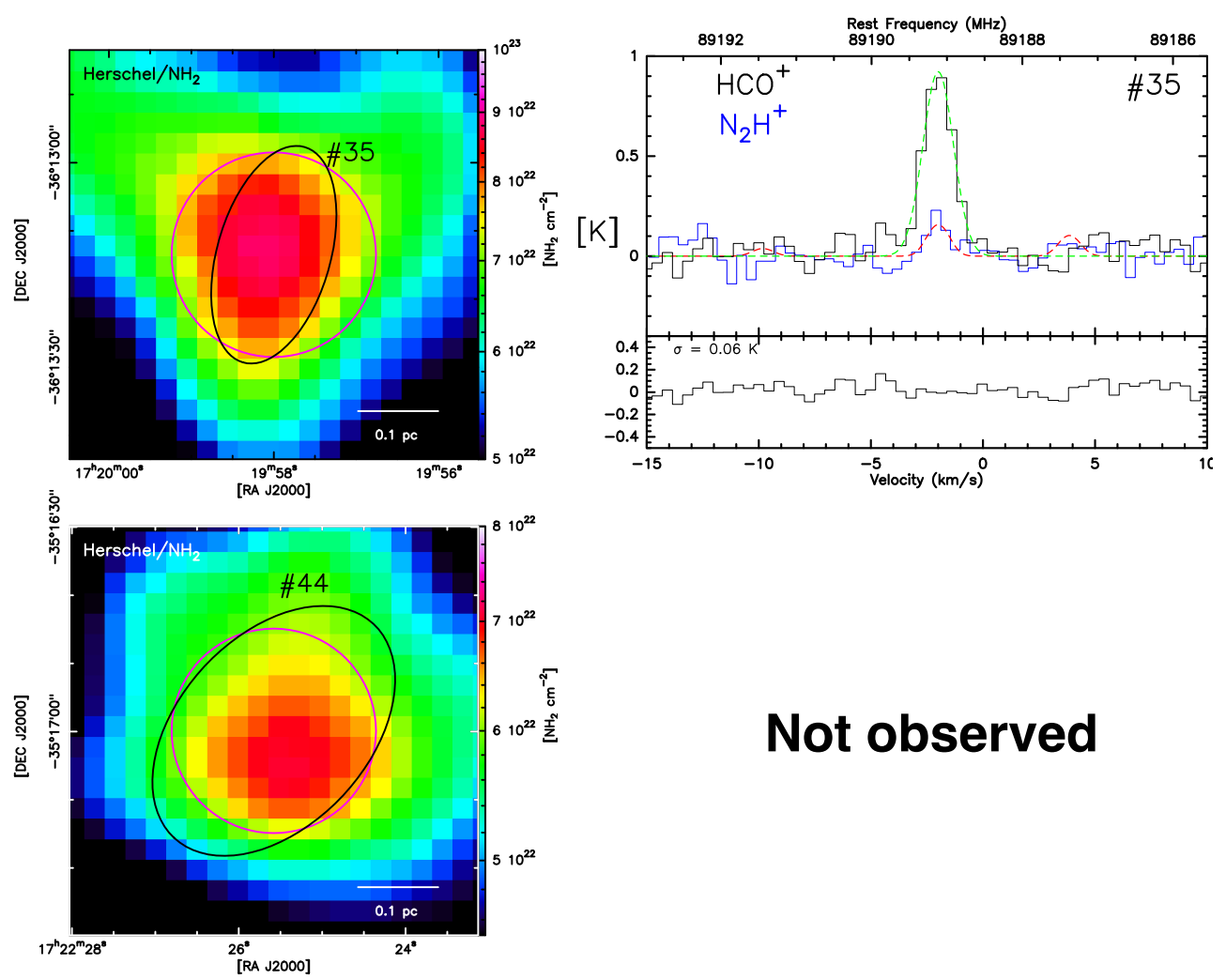

Not observed
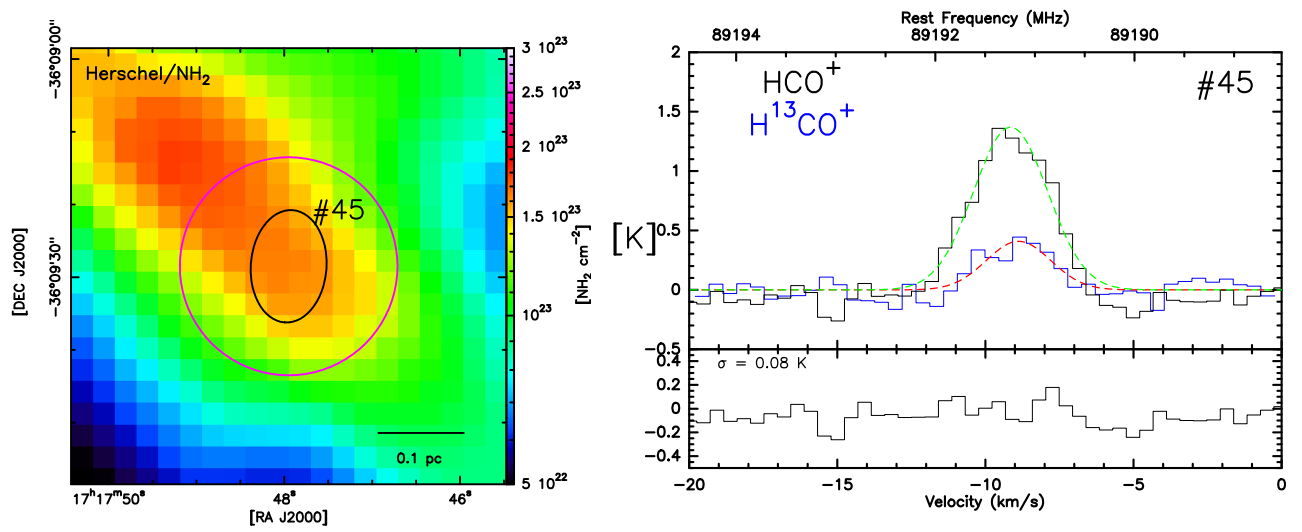

Fig. 4. Same as Fig. 2, for the MDCs $\# 35$, \#44, and \#45.
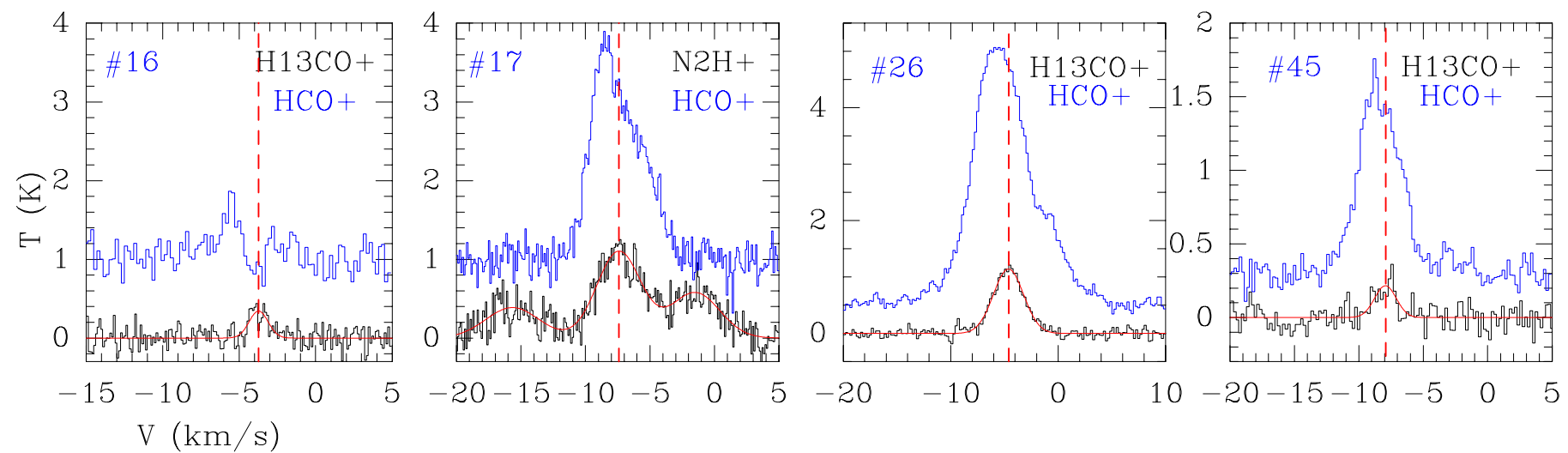

Fig. 5. Line profiles of the optically thick $\mathrm{HCO}^{+}(1-0)$ emission lines and of the optically thin $\mathrm{H}^{13} \mathrm{CO}^{+}-$respectively $\mathrm{N}_{2} \mathrm{H}^{+}(1-0)-$ emission lines toward the MDCs \#16, \#17, \#26 and \#45. The red dotted vertical line shows the velocity of the source determined from the optically thin line. 

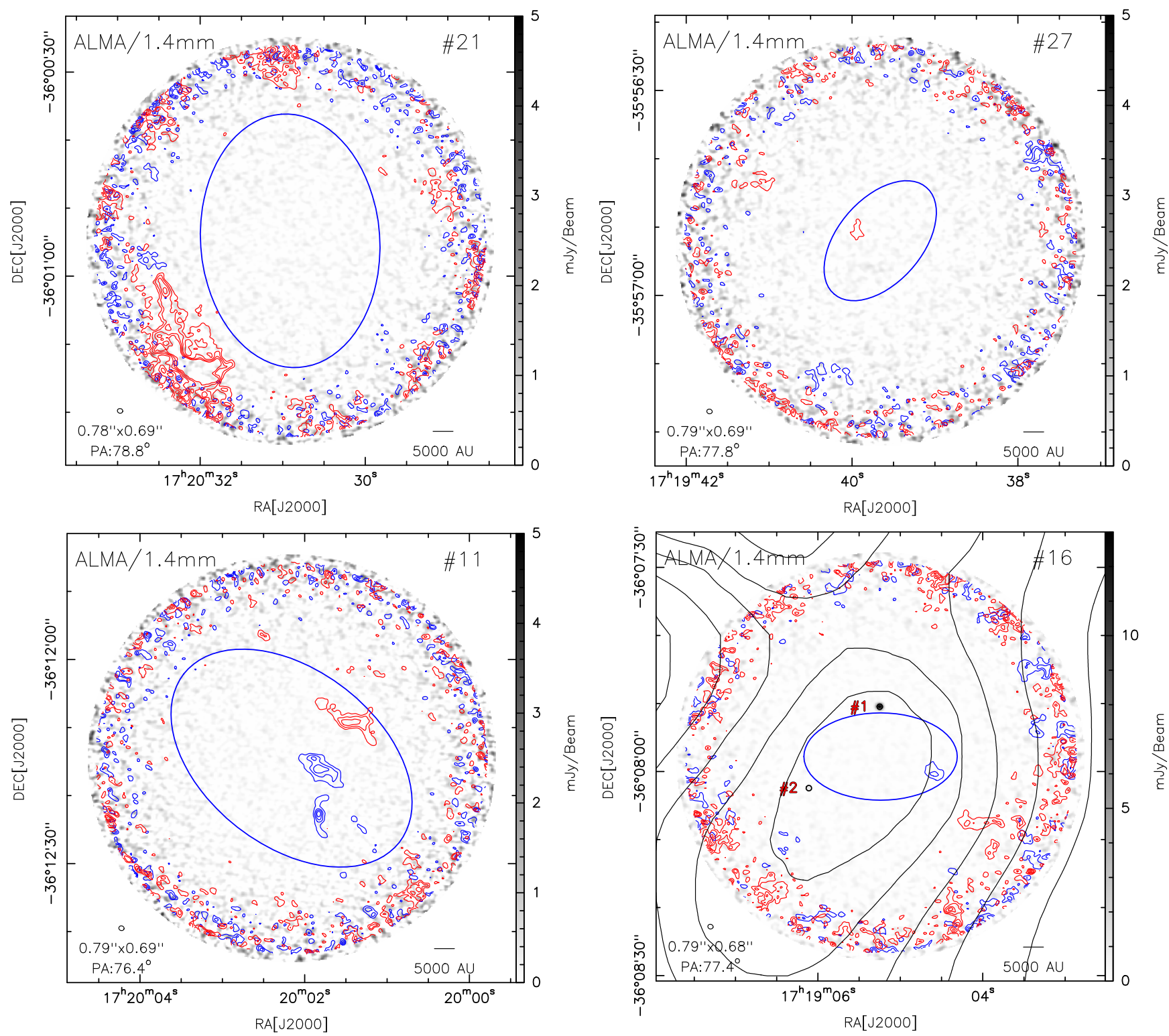

Fig. 6. ALMA 1.4 mm continuum emission toward the nine MDCs. The blue ellipses show the MDCs as defined by Tigé et al. (2017). The compact sources extracted by Getsources in the MDCs \#16, \#26, and \#45 (see text and Table 2 for details) are indicated with small black ellipses and red labels. The blue and red contours highlight the ALMA ${ }^{12} \mathrm{CO}(2-1)$ integrated emission in the blueshifted and redshifted channels, respectively. The contours start at $3 \sigma$ with a $3 \sigma$ step in each panel. The values for the integration in velocities and the resulting rms is given in Table A.2. Bottom right panel: black contours (MDC \#16) show the column density emission derived from Herschel observations (Tigé et al. 2017). The contours range from 50 to $90 \%$ by step of $10 \%$ of the peak flux in the MDC \#16 (blue ellipse). They show that the continuum sources ALMA\#1 and \#2 are associated with the MDC \#16.

hence they do not have the mass reservoir to form a high-mass star.

On the other hand the competitive accretion models (e.g. Bonnell et al. 2004) posits that an MDC of mass $M_{\mathrm{MDC}}$ will fragment into $n$ cores where each core will have a mass close to the thermal Jeans mass $\left(M_{\text {Jeans }}\right)$, and $n \simeq \frac{M_{\mathrm{MDC}}}{2.5 \times M_{\text {Jeans }}}$. From the local cloud density and kinetic temperature computed for each MDCs (see Table 1 and Tigé et al. 2017), we can calculate the Jeans mass and Jeans scale $\left(\lambda_{\text {Jeans }}\right)$ as

$M_{\text {Jeans }}=\frac{\pi^{5 / 2}}{6} \frac{\sigma_{\text {th }}^{3}}{\sqrt{\mathrm{G}^{3} \rho}}, \quad \lambda_{\text {Jeans }}=\sigma_{\text {th }} \sqrt{\frac{\pi}{\mathrm{G} \rho}}$,

where $G$ is the gravitational constant and $\rho$ is the gas density (given in Table A.1, Col. 6). $\sigma_{\text {th }}$ is the thermal broadening of the emission line given by $\sigma_{\text {th }}=\sqrt{k_{\mathrm{B}} \times T /\left(\mu \times m_{\mathrm{p}}\right)}$, where $k_{\mathrm{B}}$ is the Boltzmann constant, $\mu=2.8$ is the mean molecular weight (Kauffmann et al. 2008), $m_{\mathrm{p}}$ the mass of a proton, and $T$ is the temperature of the MDC. We obtain a mean Jeans mass $\left\langle M_{\text {Jeans }}\right\rangle=0.65 M_{\odot}$ and a mean Jeans length of $\left\langle\lambda_{\text {Jeans }}\right\rangle=0.03$ pc. Our ALMA data probe angular scales down to $0.74^{\prime \prime}(\sim 0.006 \mathrm{pc}$ at the distance of NGC 6334), and have a mass equivalent $5 \sigma$ detection threshold of $0.11 M_{\odot}$, hence we are fully sensitive to the Jeans mass and Jeans scale. Therefore, if a cluster of low-mass cores were forming in our MDCs we should have detected it. In the case of the MDC \#16 where we found cores associated with the mass reservoir of the MDC, a fragmentation such as predicted by the competitive accretion models would have resulted in $\sim 280$ cores of $\sim 0.2 M_{\odot}$ in mass. In contrast, we only found two cores of masses of 

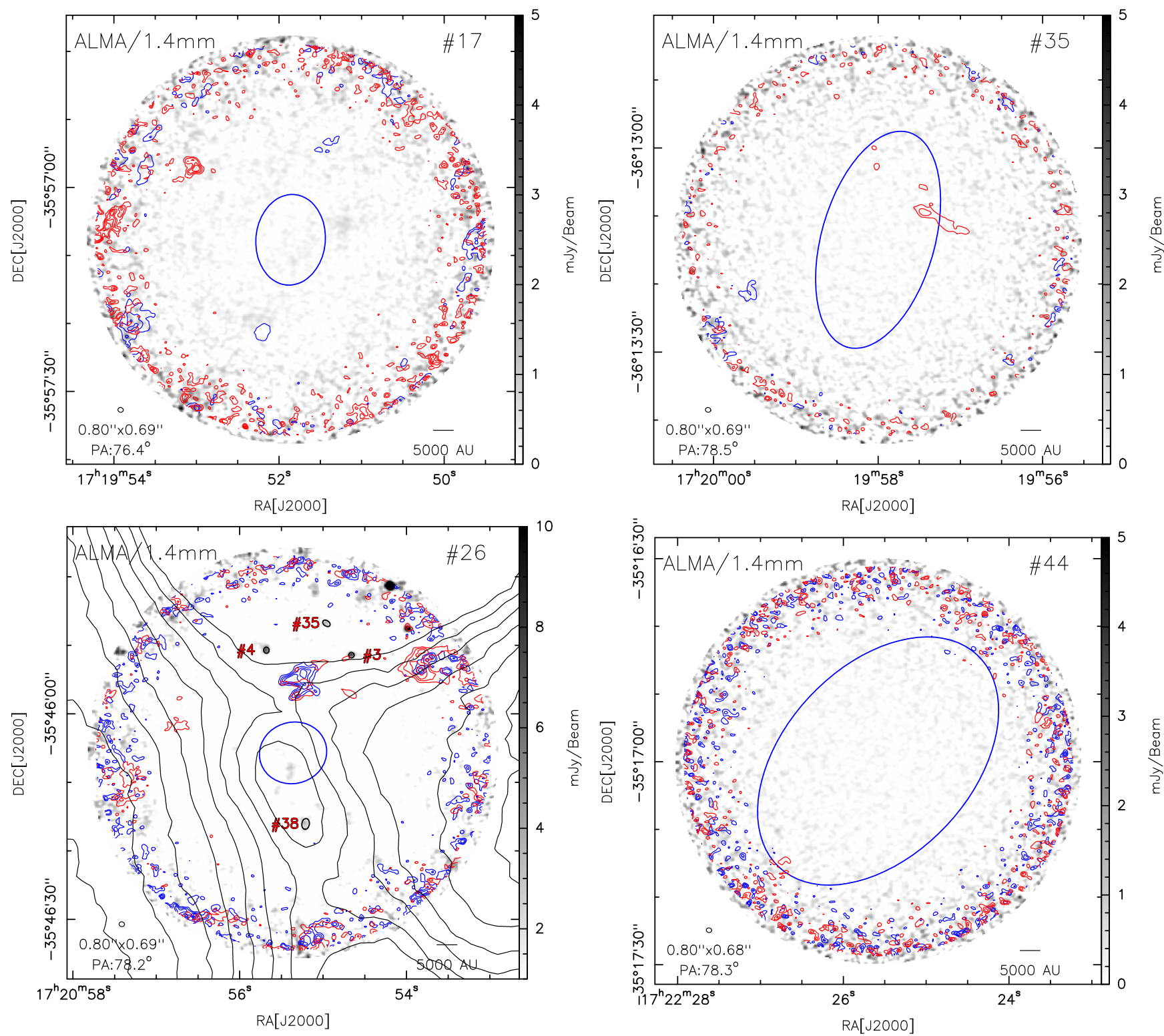

Fig. 7. Following Fig. 6. Bottom left panel: black contours show the JCMT/SCUBA-2/450 $\mu$ m emission presented in Tigé et al. (2017). The contours range from 20 to $90 \%$ of the peak flux inside the MDC \#26 (defined by the blue ellipse) by step of $10 \%$. They show that the continuum source ALMA\#38 is associated with the MDC \#26, while the continuum sources ALMA\#3, \#4, and \#35 belong to another structure.

Table 2. Physical parameters of the fragments.

\begin{tabular}{|c|c|c|c|c|c|}
\hline Source $^{a}$ & $\begin{array}{c}\text { RA } \\
(\mathrm{J} 2000)\end{array}$ & $\begin{array}{c}\text { Dec } \\
(\mathrm{J} 2000)\end{array}$ & $\begin{array}{l}\text { Integrated } \\
\text { flux (mJy) }\end{array}$ & $\begin{array}{l}\text { Size } \\
(x)\end{array}$ & $\begin{array}{c}\operatorname{Mass}^{b} \\
M_{\odot} \\
\end{array}$ \\
\hline MDC\#16-alma1 & $17: 19: 05.25$ & $-36: 07: 50.48$ & $14.2 \pm 0.1$ & $0.8 \times 0.8$ & $2.63 \pm 0.02$ \\
\hline MDC\#16-alma2 & 17:19:06.11 & $-36: 08: 02.44$ & & $0.8 \times 0.8$ & $0.17 \pm 0.02$ \\
\hline MDC\#26-alma3 & $17: 20: 54.66$ & $-35: 45: 51: 44$ & $7.1 \pm 0.6$ & $0.8 \times 0.8$ & $0.45 \pm 0.04$ \\
\hline MDC\#26-alma4 & $17: 20: 55.68$ & $-35: 45: 50.72$ & $8.8 \pm 0.5$ & $0.9 \times 0.8$ & $0.56 \pm 0.03$ \\
\hline MDC\#26-alma35 & $17: 20: 54.93$ & $-35: 45: 46.8$ & $7.0 \pm 0.9$ & $1.2 \times 0.8$ & $0.44 \pm 0.05$ \\
\hline MDC\#26-alma38 & $17: 20: 55.21$ & $-35: 46: 16: 07$ & $14.4 \pm 0.7$ & $1.6 \times 1.1$ & $0.91 \pm 0.04$ \\
\hline MDC\#45-alma1 & $17: 17: 48.59$ & $-36: 09: 11.45$ & $15.0 \pm 0.4$ & $0.8 \times 0.8$ & $1.53 \pm 0.04$ \\
\hline MDC\#45-alma2 & $17: 17: 48.66$ & $-36: 09: 34.63$ & $4.9 \pm 0.2$ & $0.8 \times 0.8$ & $0.50 \pm 0.02$ \\
\hline Mean & & & $9.0 \pm 0.4$ & $1.0 \times 0.8$ & $0.90 \pm 0.03$ \\
\hline
\end{tabular}

Notes. ${ }^{(a)}$ The label of the source is defined as the name of the nearest MDC, plus the Getsources extraction number. ${ }^{(b)}$ The method to derive the mass follows Eq. (2). 


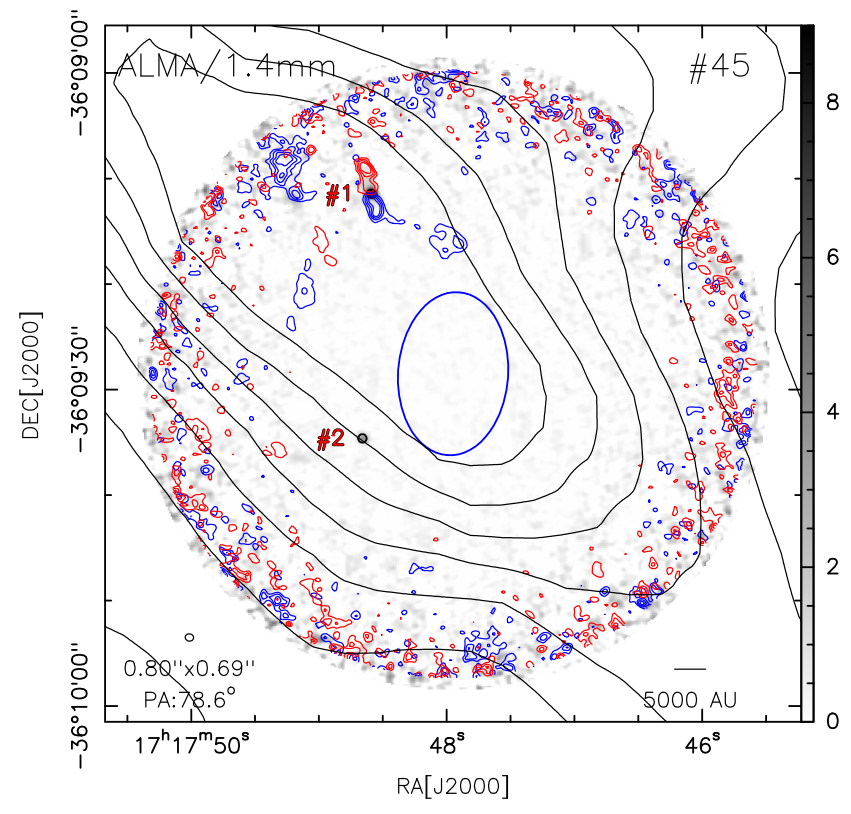

Fig. 8. Following Fig. 7. The black contours show the column density emission derived from Herschel observations (Tigé et al. 2017). The contours range from 30 to $90 \%$ of the peak flux in the MDC \#45 (blue ellipse) by step of $10 \%$. They show that the continuum sources ALMA\#1 and \#2 are not associated with the MDC \#45.

0.2 and $2.6 M_{\odot}$, respectively. In the MDC \#26 a fragmentation such as in the competitive accretion models would have resulted in $\sim 120$ cores of $\sim 0.3 M_{\odot}$ in mass, whilst we found only a single core of $\sim 0.9 M_{\odot}$ in mass. In the remaining seven MDCs no core is found associated with the starless MDCs. Therefore, the observations of starless MDCs in NGC 6334 do not match the predictions of competitive accretion models, unless the MDCs are in such early stage of evolution that the initial fragmentation has not yet taken place. Most of the mass in the starless MDCs is likely organized in extended and homogeneous cloud structures $(~ \gtrsim 0.1 \mathrm{pc})$ that were filtered by the interferometer. A similar result was reported by Dunham et al. (2016) who reported that low-mass pre-stellar cores resembling BonnortEbert spheres would be filtered out by the interferometers. Combination with short-spacing observations is therefore mandatory to investigate the exact cloud structure of starless MDCs.

It is not clear what prevents the MDCs from fragmenting into low-mass cores. According to Krumholz \& McKee (2008), the heat produced by accreting low-mass stars in regions with surface density of at least $1 \mathrm{~g} \mathrm{~cm}^{-2}$ can halt fragmentation of the MDCs. It could be the case in MDCs \#11 and \#35 if the ${ }^{12} \mathrm{CO}(2-1)$ blobs (see Sect. 3.3) were interpreted as monopolar outflows. The combination of the heat due to the accretion on the low-mass protostars with the injection of turbulence by the outflows could prevent the fragmentation in the MDCs. But the lack of fragmentation is also observed in the MDCs where no sign of proto-stellar activity is present (MDCs \#16-17-21-26-2744-45, see Figs. 6-8). Consequently, further processes must be considered.

Numerical models of a protocluster where a significant magnetic field is considered show a smaller fragmentation level than models with weak magnetic support (e.g. Commerçon et al. 2011, see also the theoretical approach by Shu et al. 1987). Therefore, the magnetic field could play the dual role of (i) limiting the fragmentation of MDCs and (ii) sustaining the MDCs against collapse together with the turbulence. Following this hypothesis, we derive the magnetic field strength necessary to prevent the MDCs from collapsing given their measured turbulence support. The magnetic field strength is computed as $B=\sigma_{A} \times \sqrt{\mu_{0} \rho}$, where $\rho$ is the mean density in the MDCs (see Table A.1), $\mu_{0}$ is the vacuum permeability and $\sigma_{A}$ is the Alfvén velocity such as $M_{\mathrm{vir}, \mathrm{B}}=M_{\mathrm{MDC}}=3 \frac{R}{G}\left(\frac{5-2 n}{3-n}\right)\left(\sigma^{2}+\frac{1}{6} \sigma_{\mathrm{A}}^{2}\right)$, where the parameters are the same as for Eq. (1). The value of the magnetic field necessary to sustain the MDCs against collapse is given in Table 1. The mean value of $\sim 1.2 \mathrm{mG}$ that we obtain is of the order of the magnetic field strength measured in high-mass star formation ( 0.1-10 mG; see Motte et al. 2018b). It is very likely that the magnetic field plays a central role in our MDCs. This is particularly true toward the MDC \#27, and \#35 where the virial parameter $\alpha_{\text {vir }}<0.4$ whereas no infall motion are traced by the molecular line emissions. This discrepancy is better explained if magnetic fields are taken into account.

\subsection{Comparison with previous observational studies}

Recently, a handset of large-sample observational studies have targeted high-mass star-forming regions with interferometers (Sanhueza et al. 2017; Kong et al. 2017; Csengeri et al. 2017). First, we note that none of these observations found a thermal Jeans fragmentation and none could claim the discovery of a high-mass pre-stellar core - in a similar way as the present study. Interestingly, these studies found different values in term of fragmentation level and/or on the masses of the cores. Here, it is primordial to remember that these studies probe different physical scales, with different filtering scales. For instance, Csengeri et al. (2017) report ALMA $7 \mathrm{~m}$ array observations that probe scales from $\sim 0.3 \mathrm{pc}$ down to $\sim 0.06 \mathrm{pc}$ in $35 \mathrm{IR}$ Quiet clumps selected from the ATLASGAL survey Schuller et al. (2009). At these scales they find that the clumps fragment on average in $3 \mathrm{MDCs}$ of typically $60 M_{\odot}$, similar to the sample we scrutinized here with ALMA, down to scales of $\sim 0.006$ pc. Csengeri et al. (2017) report that they were able to recover between $16 \%$ and $47 \%$ of the large scale fluxes of the clumps measured by the ATLASGAL survey at a similar wavelength of $870 \mu \mathrm{m}$. It is consistent with our observations that report higher filtering when probing the MDCs at higher angular resolution.

Also we stress that all these studies were conducted in different regions, hence scrutinizing different stages of the high-mass starless phase. Looking in details at these studies, they actually seem to fall well onto the empirical sequence proposed by Motte et al. (2018b). Following this sequence, (i) a starless MDC $\left(\sim 100 M_{\odot}\right.$ within $\left.0.1 \mathrm{pc}\right)$ would fragment into a few pre-stellar objects of low and/or intermediate masses, as we observed here in the MDCs \#16 and \#26. This type of fragmentation was also observed by Kong et al. (2017): they surveyed 32 high-mass surface density regions in which they detected in total 141 cores, stating that many of the weakest sources are likely to be noise fluctuation. They give the characteristics of the 50 more reliable extractions and present in details the six most massive. The six most massive have a mean mass of $3.8 M_{\odot}$ if we do not consider the most massive (C9A, see below). Therefore, all the 141 cores of Kong et al. (2017) but C9A are low or intermediate mass cores. The most massive C9A core has a mass of $\sim 70 M_{\odot}$ under their assumptions, and of $\sim 20 M_{\odot}$ using our mean temperature of $15.5 \mathrm{~K}$ and our dust emissivity assumption. Also, it could be constituted of two lower mass cores, and, its evolutionary status is unknown. (ii) Then, in the sequence of Motte et al. (2018b) the global collapse of the MDC will generate gas flows streams 
that will increase the mass of the pre-stellar objects - a process even more efficient as the pre-stellar objects are located near the gravitational potential of the MDC. This step is well supported by the observations by Sanhueza et al. (2017). They observe a candidate starless clump $\left(1500 M_{\odot}\right.$ within $\left.0.6 \mathrm{pc}\right)$ that fragments into five candidate pre-stellar cores with masses ranging from 8.4 to $15 M_{\odot}$ within $\sim 0.05$ pc. It is also supported by the present study since the two MDCs \#16 and \#26, that harbour substructure, are collapsing (see Sect. 3.2). (iii) During the accretion process, the low and/or intermediate mass pre-stellar cores eventually start proto-stellar activities, which would explain why some IR Quiet MDCs host low and/or intermediate protostars (Louvet et al., in prep.). (iv) Finally, the low and/or intermediate protostars eventually become massive $\left(\sim 25 M_{\odot}\right.$ within $0.03 \mathrm{pc}$ ) and form high-mass stars. To strengthen this view of high-mass star formation, future studies should target early-type proto-stellar MDCs $\left(\sim 100 M_{\odot}\right.$ within $\left.\sim 0.1 \mathrm{pc}\right)$ to determine the mean mass at which $0.03 \mathrm{pc}$ cores turn proto-stellar.

\section{Conclusions}

We presented $1.4 \mathrm{~mm}$ continuum and ${ }^{12} \mathrm{CO}(2-1)$ ALMA observations of nine starless massive dense cores (MDCs) located in NGC $6334(d \sim 1.75 \mathrm{kpc})$ that have a mean mass of $115 M_{\odot}$ and a mean radius of $\sim 0.1 \mathrm{pc}$. Our ALMA observations probe spatial scales from $\sim 0.1 \mathrm{pc}$ down to $0.006 \mathrm{pc}$, resolving the thermal Jeans length in these nine MDCs $\left(\left\langle\lambda_{\text {Jeans }}\right\rangle=0.03 \mathrm{pc}\right)$. Our ALMA observations are also sensitive to the thermal Jeans mass in the MDCs $\left(\left\langle M_{\text {Jeans }}\right\rangle=0.65 M_{\odot}\right)$, with a mass equivalent $5 \sigma$ level of $\sim 0.1 M_{\odot}$. We complemented the ALMA dataset with archive $\mathrm{HCO}^{+}(1-0), \mathrm{H}^{13} \mathrm{CO}^{+}(1-0)$, and $\mathrm{N}_{2} \mathrm{H}^{+}(1-0)$ MOPRA observations toward eight of the nine MDCs to examine the gas dynamic at the MDCs' scale. Our main results are summarized as follows:

- The nine starless MDCs of our sample have the potential to form a high mass star according to the mass threshold criteria presented by Baldeschi et al. (2017).

- Three MDCs are subvirialized with $\alpha_{\text {vir }} \sim 0.35$ and four MDCs are roughly virialized with $\alpha_{\text {vir }} \sim 0.8$. One among the former and three among the later MDCs further show an asymmetry of their optically thick $\mathrm{HCO}^{+}$molecular line emissions and blueshifted emission lines with respect to the optically thin $\mathrm{H}^{13} \mathrm{CO}^{+}$emission lines, indicative of collapse.

- ALMA observations show that very few 0.005 pc cores are forming in starless MDCs. In total, three cores are detected: two in the MDC \#16 with masses of $\sim 0.2 M_{\odot}$ and $2.6 M_{\odot}$ respectively, and one in the MDC \#26 with a mass of $0.9 M_{\odot}$. These three cores are deprived of outflows making them candidate pre-stellar cores. These low-mass dense cores do not have the gas reservoir to form a high-mass star. Therefore most of the gas must be organized into extended and homogeneous structures near or above our filtering scale $(\gtrsim 0.1 \mathrm{pc})$.

- These ALMA observations do not support the turbulent-core models of high-mass star formation since we do not observe high-mass pre-stellar cores (expected to have $\sim 25 M_{\odot}$ within $\sim 0.03 \mathrm{pc}$ ).

- These ALMA observations do not reproduce the predictions of the competitive accretion models in term of initial fragmentation of the MDCs. We detect only a few fragments in our sample, two orders of magnitude below the predicted fragmentation level. Our observations are therefore incompatible with these models unless the initial fragmentation has not yet taken place.
- Present ALMA observations of the starless MDCs of NGC 6334 are consistent with the empirical model by Motte et al. (2018b) in which the first stage of the high-mass star formation scenario is the formation of a few low and/or intermediate mass pre-stellar cores within the MDCs, that will grow in mass by accreting gas from the diffuse gas reservoir of the MDC.

This study shows that at the very early stages of high-mass star formation, the so-called pre-stellar MDCs have a very low fragmentation level. The fragments in these MDCs do not have the mass reservoir to form a high-mass star. Instead, the bulk of the mass of the pre-stellar MDC is composed of diffuse gas. It suggests that massive stars likely start their formation in low and/or intermediate mass pre-stellar fragments. These low and/or intermediate pre-stellar fragments would turn proto-stellar and continue accreting gas from the MDC, but also from largerscale until eventually becoming massive. Nevertheless, more follow-ups with interferometers are needed toward pre-stellar MDCs in various high-mass star forming regions to support this view.

Acknowledgements. The authors thank the anonymous referee for a thorough report which led to a significant improvement of this paper. FL acknowledges the help of Arnaud Belloche in the interpretation of optically thick $\mathrm{HCO}^{+}$emission lines. FL thanks Timea Csengeri and Sylvain Bontemps for useful discussions about the main results of the article. FL acknowledges the support of the Fondecyt program no 3170360. FL acknowledges the support of the Programme National de Physique Stellaire and Physique et Chimie du Milieu Interstellaire of CNRS/INSU (with INC/INP/IN2P3), co-funded by CEA and CNES. G.G. and L.B. acknowledges support by CONICYT project Basal AFB-170002. This paper makes use of the following ALMA data: ADS/JAO.ALMA\#2015.1.00850.S. ALMA is a partnership of ESO (representing its member states), NSF (USA) and NINS (Japan), together with NRC (Canada), NSC and ASIAA (Taiwan), and KASI (Republic of Korea), in cooperation with the Republic of Chile. The Joint ALMA Observatory is operated by ESO, AUI/NRAO and NAOJ.

\section{References}

Baldeschi, A., Elia, D., Molinari, S., et al. 2017, MNRAS, 466, 3682 Beuther, H., Linz, H., Tackenberg, J., et al. 2013, A\&A, 553, A115 Bonnell, I. A., \& Bate, M. R. 2006, MNRAS, 370, 488 Bonnell, I. A., Vine, S. G., \& Bate, M. R. 2004, MNRAS, 349, 735 Bontemps, S., Motte, F., Csengeri, T., \& Schneider, N. 2010, A\&A, 524, A18 Busquet, G., Palau, A., Estalella, R., et al. 2010, A\&A, 517, L6 Carral, P., Kurtz, S. E., Rodríguez, L. F., et al. 2002, AJ, 123, 2574 Commerçon, B., Hennebelle, P., \& Henning, T. 2011, ApJ, 742, L9 Csengeri, T., Bontemps, S., Wyrowski, F., et al. 2017, A\&A, 600, L10 Cyganowski, C. J., Brogan, C. L., Hunter, T. R., et al. 2014, ApJ, 796, L2 Duarte-Cabral, A., Bontemps, S., Motte, F., et al. 2013, A\&A, 558, A125 Duarte-Cabral, A., Bontemps, S., Motte, F., et al. 2014, A\&A, 570, A1 Dunham, M. M., Offner, S. S. R., Pineda, J. E., et al. 2016, ApJ, 823, 160 Fontani, F., Commerçon, B., Giannetti, A., et al. 2016, A\&A, 593, L14 Garay, G., Mardones, D., Brooks, K. J., Videla, L., \& Contreras, Y. 2007, ApJ, 666,309

Jackson, J. M., Rathborne, J. M., Foster, J. B., et al. 2013, PASA, 30, e057

Kauffmann, J., Bertoldi, F., Bourke, T. L., Evans, II, N. J., \& Lee, C. W. 2008, A\&A, 487, 993

Kong, S., Tan, J. C., Caselli, P., et al. 2017, ApJ, 834, 193

Kraemer, K. E., \& Jackson, J. M. 1999, ApJS, 124, 439

Krumholz, M. R., \& McKee, C. F. 2008, Nature, 451, 1082

Krumholz, M. R., Klein, R. I., \& McKee, C. F. 2007, ApJ, 656, 959

Lee, K., Looney, L. W., Schnee, S., \& Li, Z.-Y. 2013, ApJ, 772, 100 Loughran, L., McBreen, B., Fazio, G. G., et al. 1986, ApJ, 303, 629 Louvet, F., Motte, F., Hennebelle, P., et al. 2014, A\&A, 570, A15

Mardones, D., Myers, P. C., Tafalla, M., et al. 1997, ApJ, 489, 719

Matthews, H. E., McCutcheon, W. H., Kirk, H., White, G. J., \& Cohen, M. 2008, AJ, 136, 2083

McKee, C. F., \& Tan, J. C. 2002, Nature, 416, 59

Men'shchikov, A., André, P., Didelon, P., et al. 2012, A\&A, 542, A81

Motte, F., Nony, T., Louvet, F., et al. 2018a, Nat. Astron., 2, 478

Motte, F., Bontemps, S., \& Louvet, F. 2018b, ARA\&A, 56, 41

Mueller, K. E., Shirley, Y. L., Evans, II, N. J., \& Jacobson, H. R. 2002, ApJS 143,469 
Myers, P. C., Mardones, D., Tafalla, M., Williams, J. P., \& Wilner, D. J. 1996, ApJ, 465, L133

Nony, T., Louvet, F., Motte, F., et al. 2018, A\&A, 618, L5

Ossenkopf, V., \& Henning, T. 1994, A\&A, 291, 943

Palau, A., Ballesteros-Paredes, J., Vázquez-Semadeni, E., et al. 2015, MNRAS, 453, 3785

Palau, A., Zapata, L. A., Román-Zúñiga, C. G., et al. 2018, ApJ, 855, 24

Persi, P., \& Tapia, M. 2008, Handbook of Star Forming Regions, Volume II: The Southern Sky ASP Monograph Publications, ed. B. Reipurth (San Francisco, CA: ASP), 5, 456

Pillai, T., Kauffmann, J., Wyrowski, F., et al. 2011, A\&A, 530, A118

Rathborne, J. M., Simon, R., \& Jackson, J. M. 2007, ApJ, 662, 1082

Russeil, D., Zavagno, A., Motte, F., et al. 2010, A\&A, 515, A55

Russeil, D., Zavagno, A., Adami, C., et al. 2012, A\&A, 538, A142
Sanhueza, P., Jackson, J. M., Zhang, Q., et al. 2017, ApJ, 841, 97 Schuller, F., Menten, K. M., Contreras, Y., et al. 2009, A\&A, 504, 415 Shu, F. H., Adams, F. C., \& Lizano, S. 1987, ARA\&A, 25, 23 Swift, J. J. 2009, ApJ, 705, 1456

Tan, J. C., Kong, S., Butler, M. J., Caselli, P., \& Fontani, F. 2013, ApJ, 779, 96

Tigé, J., Motte, F., Russeil, D., et al. 2017, A\&A, 602, A77 Wang, K., Zhang, Q., Wu, Y., \& Zhang, H. 2011, ApJ, 735, 64 Wang, K., Zhang, Q., Testi, L., et al. 2014, MNRAS, 439, 3275 Willis, S., Marengo, M., Allen, L., et al. 2013, ApJ, 778, 96 Zhang, B., Moscadelli, L., Sato, M., et al. 2014a, ApJ, 781, 89 Zhang, Q., Qiu, K., Girart, J. M., et al. 2014b, ApJ, 792, 116 Zhang, Q., \& Wang, K. 2011, ApJ, 733, 26

Zhang, Q., Wang, Y., Pillai, T., \& Rathborne, J. 2009, ApJ, 696, 268

\section{Appendix A: Additional tables}

Table A.1. Additional parameters of the MDCs.

\begin{tabular}{|c|c|c|c|c|c|c|c|c|c|}
\hline \multicolumn{7}{|c|}{ Data set from Tigé et al. (2017) } & \multicolumn{3}{|c|}{ ALMA-cycle 3 observations } \\
\hline Source ${ }^{a}$ & $\begin{array}{c}\text { RA } \\
(\mathrm{J} 2000)\end{array}$ & $\begin{array}{c}\text { Dec } \\
(\mathrm{J} 2000)\end{array}$ & $\begin{array}{l}L_{\text {bol }}^{a} \\
\left(L_{\odot}\right)\end{array}$ & $\begin{array}{c}M / L \\
\left(M_{\odot} L_{\odot}^{-1}\right)\end{array}$ & $\begin{array}{l}\left\langle n_{\mathrm{H}_{2}}\right\rangle^{a} \\
\left(\mathrm{~cm}^{-3}\right)\end{array}$ & $\begin{array}{l}T^{a} \\
(\mathrm{~K})\end{array}$ & $\begin{array}{c}\sigma_{12}^{b} \mathrm{CO} \\
\left(\mathrm{mJy} \mathrm{beam}^{-1}\right)\end{array}$ & $\begin{array}{c}\sigma_{\text {cont }} \\
\left(\mu \mathrm{Jy} \mathrm{beam}^{-1}\right)\end{array}$ & $\begin{array}{c}\text { Mass sensitivity } \\
\text { at } 5 \sigma^{c}\end{array}$ \\
\hline NGC 6334-MDC\#11 & $17: 20: 02.16$ & $-36: 12: 14.5$ & 110 & 1.55 & $2.4 \times 10^{5}$ & 14.1 & 15.8 & 273 & $0.11 M_{\odot}$ \\
\hline NGC 6334-MDC\#16 & 17:19:05.24 & $-36: 07: 57.8$ & 11 & 13.64 & $1.6 \times 10^{6}$ & 9.9 & 15.8 & 263 & $0.19 M_{\odot}$ \\
\hline NGC 6334-MDC\#17 & $17: 19: 51.86$ & $-35: 57: 07.7$ & 230 & 0.65 & $4.8 \times 10^{6}$ & 16.5 & 16.8 & 310 & $0.10 M_{\odot}$ \\
\hline NGC 6334-MDC\#21 & $17: 20: 30.91$ & $-36: 00: 54.8$ & 390 & 0.33 & $2.2 \times 10^{5}$ & 18.3 & 16.9 & 283 & $0.06 M_{\odot}$ \\
\hline NGC 6334-MDC\#27 & $17: 19: 39.66$ & $-35: 56: 52.0$ & 120 & 0.88 & $1.3 \times 10^{6}$ & 15.6 & 16.4 & 292 & $0.07 M_{\odot}$ \\
\hline NGC 6334-MDC\#26 & $17: 20: 55.36$ & $-35: 46: 05.7$ & 740 & 0.14 & $7.5 \times 10^{6}$ & 21.1 & 17.6 & 656 & $0.16 M_{\odot}$ \\
\hline NGC 6334-MDC\#35 & $17: 19: 58.00$ & $-36: 13: 13.5$ & 140 & 0.64 & $3.4 \times 10^{5}$ & 16.4 & 17.2 & 318 & $0.11 M_{\odot}$ \\
\hline NGC 6334-MDC\#44 & $17: 22: 25.58$ & $-35: 16: 59.9$ & 27 & 2.85 & $1.0 \times 10^{5}$ & 12.8 & 16.5 & 281 & $0.08 M_{\odot}$ \\
\hline NGC 6334-MDC\#45 & $17: 17: 47.95$ & $-36: 09: 28.5$ & 60 & 1.27 & $2.0 \times 10^{6}$ & 14.7 & 16.5 & 352 & $0.14 M_{\odot}$ \\
\hline Mean & - & - & 203 & 2.44 & $2.0 \times 10^{6}$ & 15.5 & 16.6 & 336 & $0.11 M_{\odot}$ \\
\hline
\end{tabular}

Notes. ${ }^{(a)}$ The source's name, radius, bolometric luminosity, and density follow the work by Tigé et al. (2017). ${ }^{(b)}$ The $1 \sigma$ rms noise level is given per channel, where the channel width is $0.7 \mathrm{~km} \mathrm{~s}^{-1}$. ${ }^{(c)}$ The method to estimate the mass from the $5 \sigma$ value follows Eq. (2).

Table A.2. Integration parameters of the CO line in the MDCs.

\begin{tabular}{lccccc}
\hline \hline MDC & \multicolumn{2}{c}{ Blueshifted ${ }^{12} \mathrm{CO}(2-1)$ emission } & & \multicolumn{2}{c}{ Redshifted ${ }^{12} \mathrm{CO}(2-1)$ emission } \\
\cline { 2 - 3 } & $\begin{array}{c}\text { Velocity range } \\
\left(\mathrm{km} \mathrm{s}^{-1}\right)\end{array}$ & $\begin{array}{c}1 \sigma \text { value } \\
\left(\mathrm{mJy} \mathrm{beam}^{-1} \mathrm{~km} \mathrm{~s}^{-1}\right)\end{array}$ & $\begin{array}{c}\text { Velocity range } \\
\left(\mathrm{km} \mathrm{s}^{-1}\right)\end{array}$ & $\begin{array}{c}1 \sigma \text { value } \\
\left(\mathrm{mJy} \mathrm{beam}^{-1} \mathrm{~km} \mathrm{~s}^{-1}\right)\end{array}$ \\
\hline NGC 6334-MDC\#11 & {$[-19.4 ;-7.9]$} & 11.1 & & {$[0.0 ; 3.1]$} & 6.2 \\
NGC 6334-MDC\#16 & {$[-8.5 ;-5.5]$} & 11.9 & & {$[1.0 ; 6.1]$} & 7.3 \\
NGC 6334-MDC\#17 & {$[-13.3 ;-8.9]$} & 20.1 & & {$[-0.1 ; 5.7]$} & 5.7 \\
NGC 6334-MDC\#21 & {$[-12.6 ;-9.7]$} & 4.5 & & {$[10.9 ; 14.2]$} & 7.9 \\
NGC 6334-MDC\#27 & {$[-18.6 ;-9.3]$} & 8.2 & & {$[-2.2 ; 6.4]$} & 9.3 \\
NGC 6334-MDC\#26 & {$[-30.1 ;-20.5]$} & 7.8 & & {$[-7.9 ;-5.3]$} & 15.9 \\
NGC 6334-MDC\#35 & {$[-9.8 ;-4.5]$} & 7.4 & & {$[1.8 ; 5.4]$} & 4.8 \\
NGC 6334-MDC\#44 & {$[-16.5 ;-7.5]$} & 6.3 & & {$[1.5 ; 10.7]$} & 6.6 \\
NGC 6334-MDC\#45 & {$[-23.3 ;-17.0]$} & 9.6 & {$[0.5 ; 13.3]$} & 9.9 \\
\hline
\end{tabular}

